\title{
PET Evidence for a Role for Striatal Dopamine in the Attentional Blink: Functional Implications
}

\author{
Heleen A. Slagter ${ }^{1}$, Rachel Tomer ${ }^{2}$, Bradley T. Christian ${ }^{3}$, \\ Andrew S. Fox ${ }^{3}$, Lorenza S. Colzato ${ }^{4}$, Carlye R. King ${ }^{3}$, \\ Dhanabalan Murali ${ }^{3}$, and Richard J. Davidson ${ }^{3}$
}

\begin{abstract}
Our outside world changes continuously, for example, when driving through traffic. An important question is how our brain deals with this constant barrage of rapidly changing sensory input and flexibly selects only newly goal-relevant information for further capacity-limited processing in working memory. The challenge our brain faces is experimentally captured by the attentional blink (AB): an impairment in detecting the second of two target stimuli presented in close temporal proximity among distracters. Many theories have been proposed to explain this deficit in processing goal-relevant information, with some attributing the $\mathrm{AB}$ to capacity limitations related to encoding of the first target and others assigning a critical role to on-line selection mechanisms that control access to working memory. The current study examined the role of striatal dopa-
\end{abstract}

\section{INTRODUCTION}

In an ever-changing world, our senses are continuously bombarded with more information than our brain can process up to the level of awareness. Hence, the ability to rapidly select goal-relevant information when it occurs and inhibit irrelevant or distracting information is central to goal-directed behavior. The challenge our brain faces when presented with an overwhelming amount of information to analyze is captured by one of the most studied attentional phenomena in the literature: the so-called attentional blink (AB) deficit (Raymond, Shapiro, \& Arnell, 1992). This deficit occurs when people have to detect two target stimuli (T1 and T2) presented in close temporal succession and among distracter events. Specifically, when T2 follows T1 within 100-500 msec, it often goes unnoticed. Different models have been proposed to explain this deficit in selective information processing, with many early models attributing the $\mathrm{AB}$ to limitations in processing capacity (Shapiro, Raymond, \& Arnell, 1997). The general idea underlying these models (e.g., Chun \& Potter, 1995) is that, to become available for conscious report, a stimulus

\footnotetext{
${ }^{1}$ University of Amsterdam, ${ }^{2}$ University of Haifa, ${ }^{3}$ University of
} Wisconsin, ${ }^{4}$ Leiden University mine in the $\mathrm{AB}$, given its known role in regulating the contents of working memory. Specifically, participants performed an $\mathrm{AB}$ task and their basal level of dopamine D2-like receptor binding was measured using PET and [F-18]fallypride. As predicted, individual differences analyses showed that greater D2-like receptor binding in the striatum was associated with a larger $\mathrm{AB}$, implicating striatal dopamine and mechanisms that control access to working memory in the AB. Specifically, we propose that striatal dopamine may determine the $\mathrm{AB}$ by regulating the threshold for working memory updating, providing a testable physiological basis for this deficit in gating rapidly changing visual information. A challenge for current models of the $\mathrm{AB}$ lies in connecting more directly to these neurobiological data.

has to be encoded and consolidated in working memory, a process that is assumed to consume limited processing recourses. If these resources are allocated to consolidation of T1, fewer resources are available for T2, rendering the sensory representation of $\mathrm{T} 2$ more susceptible to decay and interruption by distracters, so that $\mathrm{T} 2$ is less likely to be maintained and reported later.

Yet, limited-capacity models have difficulty explaining recent behavioral findings showing that individuals can easily process up to four targets presented in close temporal proximity, as long as there is no intervening distracter stimulus (Di Lollo, Kawahara, Shahab Ghorashi, \& Enns, 2005). This observation, as well as others (e.g., Olivers, van der Stigchel, \& Hulleman, 2007; Kawahara, Kumada, \& Di Lollo, 2006), have stimulated the development of novel theories that posit that the $\mathrm{AB}$ results from on-line selection mechanisms that act in response to distracting input rather than being the result of T1-induced cognitive resource depletion (for recent reviews, see Martens \& Wyble, 2010; Dux \& Marois, 2009). For example, it has been postulated that individuals apply an attentional set (or filter) for targets and against distracters and that the $\mathrm{AB}$ results from disruption of the input control filter by $\mathrm{T} 1$ processing (Di Lollo et al., 2005) or an inhibitory response meant to suppress D1, but which accidentally 
suppresses the subsequently presented T2 (Olivers \& Meeter, 2008).

Thus far, the majority of $\mathrm{AB}$ accounts make inferences about the exact processes that contribute to the $\mathrm{AB}$ solely based on the indirect measure of performance. Studies examining the neurobiological mechanisms underlying the $\mathrm{AB}$ may provide additional information critical for understanding this deficit in gating rapidly changing visual information. The current study examined the role of striatal dopamine in the $\mathrm{AB}$ given its known role in controlling information flow into working memory (e.g., Cools \& D'Esposito, 2011; Frank, Loughry, \& O'Reilly, 2001; Braver \& Cohen, 1999). Many AB theories emphasize the importance of working memory for consolidating target information (Martens \& Wyble, 2010; Dux \& Marois, 2009). Indeed, behavioral work has shown that the size of the $\mathrm{AB}$ is related to operating resources within working memory, including one's ability to keep irrelevant information out of working memory (Arnell, Stokes, MacLean, \& Gicante, 2010; Arnell \& Stubitz, 2010; Martens \& Valchev, 2009; Dux \& Marois, 2008; Colzato, Spapé, Pannebakker, \& Hommel, 2007). Notably, a large body of research has linked working memory operations to interactions between the striatum and frontal cortex and has revealed a critical role for the neurotransmitter dopamine in regulating these interactions. In particular, whereas frontal dopamine appears critical for maintaining working memory representations and thus stability of behavior, striatal dopamine is thought to be critical for the updating of frontal working memory representations and hence, flexibility of behavior (e.g., Cools \& D'Esposito, 2011; Frank et al., 2001; Braver \& Cohen, 1999). Indeed, striatal dopamine has been shown to promote cognitive flexibility by allowing updating of newly relevant representations in working memory and preventing gating of nonrelevant representations (e.g., Wang, Vijayraghavan, \& Goldman-Rakic, 2004; Frank et al., 2001). On the basis of this work, we previously hypothesized that striatal dopamine may play an important role in the $\mathrm{AB}$ by regulating which stimuli enter working memory (Colzato, Slagter, de Rover, \& Hommel, 2011; Colzato, Slagter, Spapé, \& Hommel, 2008). Consistent with this hypothesis, we found that individual $\mathrm{AB}$ size is predicted by spontaneous eye blink rate, a marker of striatal dopaminergic functioning (Karson, 1983), and by the C957T polymorphism at the DRD2 gene (associated with striatal D2 receptors; Colzato et al., 2008, 2011). These observations provide preliminary, albeit indirect, evidence for a role of striatal dopamine in the $\mathrm{AB}$.

The current study tested the hypothesis that striatal dopamine and in particular striatal D2 receptors play a role in the $\mathrm{AB}$ more directly. To this end, participants took part in three sessions: (1) an AB task session, (2) a PET session in which baseline D2-like (D2/D3) receptor availability was measured using the ligand [F-18] fallypride at rest, and (3) an MRI session for anatomical localization purposes. Our main prediction was that individual variation in striatal D2-like receptor binding would predict individual $\mathrm{AB}$ size.
Notably, D2 receptors are expressed primarily in the striatal indirect pathway (Gerfen, 1992), which is thought to prevent gating of irrelevant representations into working memory (Hazy, Frank, \& O'Reilly, 2007a; O'Reilly \& Frank, 2006). An association between striatal D2-like receptor binding and $\mathrm{AB}$ size would thus indicate that striatal dopaminergic functioning may represent an important neurobiological mechanism underlying distracter suppression efficiency and hence the ability to rapidly and flexibly gate only newly relevant information into working memory for further processing in the $\mathrm{AB}$ task.

\section{METHODS}

\section{Participants}

Fourteen right-handed adults (nine women, 19-29 years, mean age $=20.2$ years) participated in this study. Only healthy participants without history of developmental disorders, head trauma, psychiatric or neurological disease, and drug abuse were included. All had normal or corrected-to-normal visual acuity. Participants were asked to abstain from eating, drinking (other than water), and smoking for minimally $4 \mathrm{hr}$ before the PET session. They were compensated for participation. The study was approved by the local ethics committee.

\section{Experimental Design and Procedure}

Data were collected in three sessions, separated by 14 weeks: a behavioral session, a PET imaging session, and an MRI session.

\section{Behavioral Session}

Participants performed an $\mathrm{AB}$ task adopted from Slagter et al. (Slagter, Lutz, Greischar, Nieuwenhuis, \& Davidson, 2009; Slagter et al., 2007). They had to identify two digits presented in a rapid stream of letters (Figure 1). Each trial started with a fixation-plus sign $(+; 2000 \mathrm{msec})$. After a blank interval $(250 \mathrm{msec})$, the stimulus stream began, consisting of 20 items, each presented in black on a gray background (red, green, blue: 70, 70, 70) at the center of the screen (16 point Times New Roman) for $67 \mathrm{msec}$, followed by a 33-msec blank. Each letter was randomly drawn (without replacement) from the alphabet (except $\mathrm{I}, \mathrm{O}, \mathrm{S}$, and Z). Digits were drawn randomly from the set 1-9. T1 position was varied randomly between 7 and 9 . T2 was presented directly thereafter (Lag 1) or after two, four, or seven distracters (at Lags 3, 5, or 8, respectively). Previous work using a highly similar task has shown a large $\mathrm{AB}$ at Lag 3 compared with Lag 8 position (Colzato et al., 2008, 2011). Both targets were to be reported $250 \mathrm{msec}$ after the stream by pressing the corresponding digit keys. Participants first practiced the task for 24 trials. Then, they performed two blocks of 48 trials each, resulting in 24 trials per lag condition, all intermixed within blocks. 


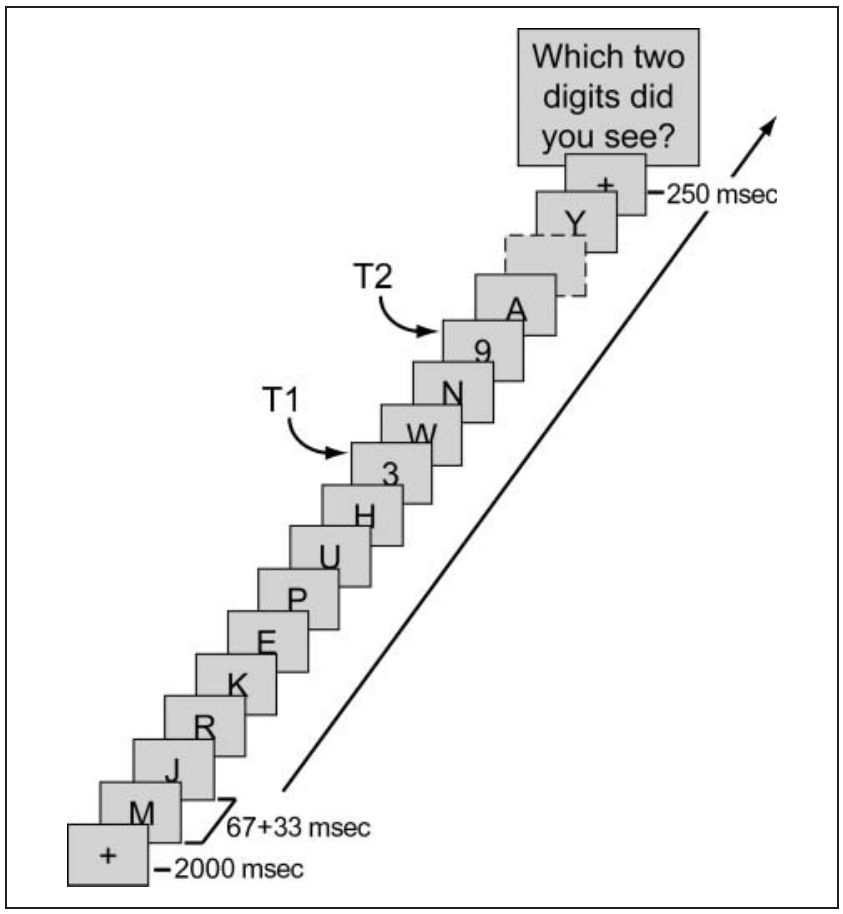

Figure 1. The attentional blink task. Participants had to detect two targets (digits) in a rapid stream of distracters (letters). T2 followed T1 at Lags 1, 3, 5, or 8.

\section{PET Data Acquisition Session}

PET data were acquired using a Siemens EXACT HR+ PET scanner in 3-D mode (septa retracted) and the highaffinity D2/D3 radioligand [F-18] fallypride. Individual differences in D2-like receptor availability as measured by [F-18] fallypride are stable across time (Mukherjee et al., 2002) and thus provide an index of stable individual differences in striatal dopaminergic neurotransmission. [F-18] fallypride expresses the highest affinity for D2 receptors, but also binds to D3 receptors and (albeit weakly) to D4 receptors. The subjects were positioned head first, supine with the cantho-meatal line parallel to the in-plane field of view. The head rests in the scanner head holder extending from the patient bed and held in place by surgical tape placed firmly across the subject's forehead. A 5-min transmission scan was then acquired to correct for the attenuation of the gamma rays within the tissue. The acquisition of the dynamic [F-18] fallypride PET scan was initiated with the injection of radioligand $(237 \pm 43 \mathrm{MBq})$. The synthesis of [F-18] fallypride was carried out using previously reported methods (Mukherjee, Yang, Das, \& Brown, 1995). The final sterile $0.9 \%$ saline solution of [F-18] fallypride was produced with radiochemical purity greater than 95\% and specific activity of $227 \pm 140 \mathrm{GBq} / \mathrm{micromol}$. A 150-min dynamic acquisition was acquired, initiating with the 30-sec bolus infusion of radiotracer. The time series were binned into six 1-min frames and 48 3-min frames.

\section{MRI Data Acquisition Session}

Anatomical brain images were acquired on a 3T-GE Signa scanner, equipped with high-speed gradients and a wholehead transmit-receive quadrature birdcage headcoil (GE Medical Systems). Anatomical scans consisted of a highresolution 3-D T1-weighted, inversion-recovery fastgradient-echo image (inversion time $=600 \mathrm{msec}$, in-plane resolution $=256 \times 256$, field of view $=240 \mathrm{~mm}, 124 \times$ $1.1 \mathrm{~mm}$ axial slices), and a T2-weighted, fast-spin echo image (in-plane resolution $=256 \times 256$, field of view $=$ $240 \mathrm{~mm}, 81 \times 2 \mathrm{~mm}$ sagittal slices).

\section{Data Analysis \\ Behavioral Data Analysis}

T1 and T2 accuracy data were submitted to separate repeated-measures ANOVAs with Lags (1, 3, 5, and 8) as a within-subject factor. T2 accuracy was based only on those trials in which $\mathrm{T} 1$ was correctly reported.

\section{Brain Imaging Data Analysis}

PET data were reconstructed using a filtered back-projection algorithm with sinogram trimming; axial and in-plane smoothing ( $4 \mathrm{~mm}$ Gaussian filter) to a voxel size of $1.84 \mathrm{~mm} \times 1.84 \mathrm{~mm} \times 2.43 \mathrm{~mm}$; and corrected for random events, annihilation radiation attenuation, deadtime, scanner normalization, and scatter radiation. The reconstructed PET time series was then inspected and corrected for head motion using the SPM2 coregistration (www.fil. ion.ucl.ac.uk/spm) algorithm based on frame-to-frame coregistration to an early integrated reference image. The cerebellar time-activity curve was extracted from the PET data based on an ROI drawn on the cerebellar lobes of the early PET data. Parametric images of distribution volume ratios (DVRs) were generated using this time course to represent the behavior of the radiotracer in brain regions with negligible binding (Mukherjee et al., 2002). The DVR parameter represents an index that is proportional to the concentration of available D2-like binding sites $\left(B_{\max }\right)$, given by the relationship:

$$
\mathrm{DVR}=\left(B_{\max } / K_{\mathrm{D}}\right) f_{\mathrm{ND}}+1
$$

where $K_{\mathrm{D}}$ is the apparent (in vivo) equilibrium dissociation constant and $f_{\mathrm{ND}}$ is the free fraction of radiotracer in the brain tissue (Innis et al., 2007). A multilinear approach was used to generate the DVR estimates using the data starting at $39 \mathrm{~min}\left(t^{*}\right)$ until the end of the acquisition (Ichise, Toyama, Innis, \& Carson, 2002; Logan et al., 1996). The DVR parametric images were spatially coregistered to the same participant's T1-weighted MRI images using the FMRIB Software Library (FSL) linear registration tool (Jenkinson \& Smith, 2001).

T1-weighted MRI images were manually masked to exclude nonbrain tissues. These "skull-stripped" images 
for each participant were coregistered to the MNI-152 template packaged with FSL. The resulting standard-space, skull-stripped T1-weigted MRI images were averaged to create single-participant templates in standard space. Small differences in brain morphology were accounted for by performing a nonlinear warp using FSL's nonlinear registration tool (www.fmrib.ox.ac.uk/analysis/techrep/ tr07ja2/tr07ja2.pdf) to align each participant's standard-space skull-stripped T1-weighted MRI images to their single-subject template. These transformations were then combined with the DVR to T1-weighted MRI transforms and applied to the DVR images to create binding images. Because differences observed in across-subject analyses might result from individual differences in brain anatomy (Woodward et al., 2009; Oakes et al., 2007) rather than true differences in binding, we assessed the probability of gray matter (GMP) at each voxel in each participant's brain based on the skull-stripped standard-space T1-weighted MRIs using FSL's automated segmentation tool (Zhang, Brady, \& Smith, 2001). Before statistical analyses, all images were blurred using an 8-mm FWHM Gaussian filter to account for potential across participant differences in anatomy.

Our main prediction was that striatal D2-like receptor binding would be predictive of individual differences in $\mathrm{AB}$ size, defined as the difference in $\mathrm{T} 2$ accuracy between Lag 8 and Lag 3 trials. To examine this prediction, we correlated individual differences in receptor binding with individual differences in $\mathrm{AB}$ size (while controlling for GMP at each voxel) using Spearman's rank correlation). Only voxels with an average dopamine binding value of 1.2 or greater (i.e., greater than 1.2 times the cerebellar binding rate) were included in the correlation analysis to exclude most white matter regions. Significance testing was carried out via a two-stage permutation testing (Nichols \& Holmes, 2002). At the first stage (voxel level), as a first step, GMP was regressed out of D2-like receptor binding. Then, participant identity was randomly shuffled, and the Spearman's correlation between $\mathrm{AB}$ size and the residualized binding values was computed again at each voxel. This was repeated 1,000 times, generating a distribution of correlation coefficients at each voxel under the null hypothesis of no relationship between $\mathrm{AB}$ size and D2-like receptor binding (while controlling for GMP). Statistical $Z$ values were taken as the normalized distance of the real correlation coefficient compared with the null distribution. Voxels with a $Z$ value greater than $2.6(p<.005)$ were retained as being significant at the voxel level. In the second stage (cluster level), Z values were computed based on one of the 1,000 random permutation iterations, and the statistical map was thresholded again. This time, the number of voxels in the largest suprathreshold cluster was stored. This was repeated 500 times, generating a distribution of maximum cluster sizes under the null hypothesis. The cluster threshold was defined as the standardized distance from the mean of the maximum cluster distribution corresponding to $p<.05$ (20 contiguous voxels).

\section{RESULTS}

\section{Behavioral Results}

As expected and shown in Figure 2, participants exhibited a marked $\mathrm{AB}$, with lower performance at Lag 3 than at Lags 1,5 , and 8 (main effect of Lag: $F(3,39)=3.6, p=$ .022). This pattern was confirmed by post hoc polynomial contrasts revealing a significant cubic trend, $F(1,13)=4.8$, $p=.046$. Furthermore, in line with the literature (e.g., Martens, Munneke, Smid, \& Johnson, 2006), there was large variability among participants in the size of the $\mathrm{AB}$, as indexed by the difference in T2 accuracy between Lags 8 and 3 . AB size varied across individuals between $-7.8 \%$ and $40.7 \%$. T1 accuracy did not differ significantly between lags, $F(3,39)<1, p=.69$. T1 accuracy was overall high: 99\%, 99\%, 100\%, and 99\% at Lags 1, 3, 5, and 8, respectively.

\section{D2-like Receptor Binding and AB Size}

Our main prediction was that individual variation in $\mathrm{AB}$ size would be associated with individual variation in striatal D2-like receptor binding, as measured in a separate session using PET and the radioligand [F-18] fallypride during resting conditions. Confirming this prediction, voxelwise correlation analysis revealed a significant correlationafter correction for GMP-between $\mathrm{AB}$ size and D2-like receptor binding in the dorsal striatum, bilaterally and in the vicinity of the caudate nucleus and globus pallidus (Figure 3). More specifically, in both the left and the right striatum, higher binding was associated with a larger $\mathrm{AB}$ to T2, as can be seen in Figure 4. This scatter plot displays the correlation between $\mathrm{AB}$ size and binding for the voxel showing the highest correlation for each striatal cluster separately. The $x y z$ MNI coordinates of the voxel showing

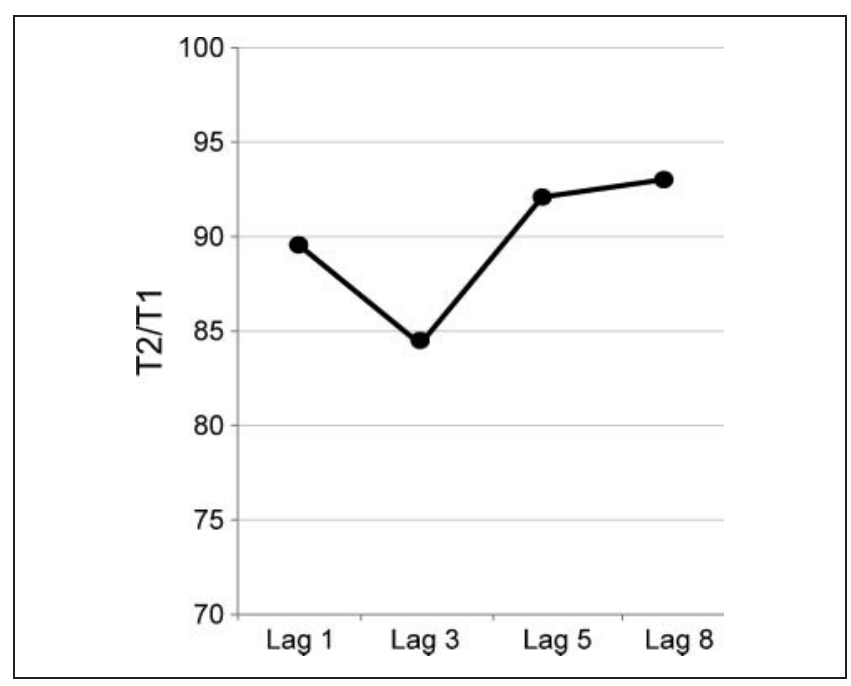

Figure 2. AB task performance. Average detection rates of $\mathrm{T} 2$ (contingent on T1 detection) are displayed as a function of lag. This figure shows a marked decrease in $\mathrm{T} 2$ detection rate at Lag 3, that is, an AB. 


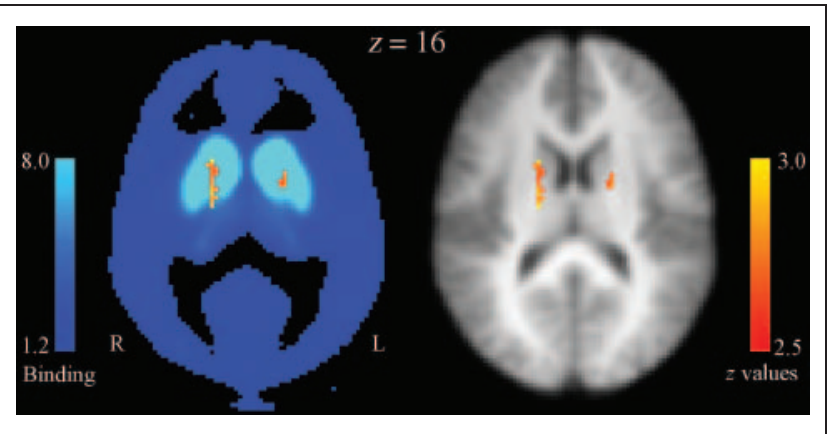

Figure 3. Regions of the striatum showing a significant relationship between D2-like receptor binding and individual AB size. Displayed is the axial section $(z=16)$ of the statistical map of the correlation between individual [F-18]fallypride binding and $\mathrm{AB}$ size overlaid upon (on the left) the group-averaged binding map and (on the right) the average MRI of all participants in MNI space.

the highest correlation $(r(12)=.70, p=.005)$ within the left striatal cluster were: $-16,-2,18$. The right striatal cluster contained two peaks. The $x y z$ MNI coordinates of the first peak $(r(12)=.81, p<.001)$ were $18,-8,18$, and the $x y z$ MNI coordinates of the second peak $(r(12)=.71$, $p=.005$ ) were $20,12,12$.

In contrast to several other D2 radioligands (e.g., [C-11] raclopride), [F-18] fallypride provides sufficient signal-to-noise ratio to quantify D2-like binding in extrastriatal areas, such as cortex, where the concentration of D2 receptors is much lower than that in the striatum. Yet, no other brain region exhibited a relationship (negative or positive) between $\mathrm{AB}$ size and $\mathrm{D} 2$-like receptor availability, even at a more lenient voxel-level threshold of $p<.01$.

\section{DISCUSSION}

In this PET study, we examined the relationship between striatal dopamine, as indexed by baseline D2-like receptor binding, and the $\mathrm{AB}$, a deficit in processing two meaningful events presented in close temporal proximity and among distracter events. As predicted, increased D2-like receptor availability in the striatum was associated with a larger $\mathrm{AB}$ to T2. This novel finding supports the idea that striatal dopaminergic neurotransmission; hence, mechanisms involved in dynamically regulating the contents of working memory play an important role in the $\mathrm{AB}$. It corroborates previous, more indirect evidence implicating the striatum, and in particular the striatal D2 pathway, in the $\mathrm{AB}$ (Colzato et al., 2008, 2011; Slagter, Johnstone, Beets, \& Davidson, 2010).

Increased D2-like receptor binding in the striatum in individuals with a relatively large $\mathrm{AB}$ may correspond to higher receptor densities or lower levels of (competing) endogenous dopamine or both. It is notable in this respect that genetic overexpression of striatal D2 receptors in rodents causes impairments in set shifting (Kellendonk et al., 2006). Furthermore, a lower basal level of endogenous striatal dopamine in individuals with a large $A B$ would be in line with our previous observation that these individuals show a relatively low spontaneous eye blink rate (sEBR), an index of striatal dopaminergic function (Colzato et al., 2008; Karson, 1983).

An important question for future research is how precisely striatal dopamine may determine the $\mathrm{AB}$. As mentioned in the Introduction, previous work has shown that high levels of striatal dopamine promote cognitive flexibility by permitting updating of frontal working memory representations, whereas low striatal dopamine levels are associated with cognitive stability and little updating in response to novel relevant information (for recent reviews, see, e.g., Cools \& D'Esposito, 2011; Maia \& Frank, 2011). Our observation that more D2-like receptor binding (hence, likely a lower level of [competing] endogenous dopamine) was generally associated with a larger $\mathrm{AB}$ is in line with the notion that low striatal dopamine levels promote stabilization of frontal task-relevant representations
Figure 4. Striatal D2-like receptor binding predicted $\mathrm{AB}$ size across participants. Correlation plots showing the significant cross-subject relationship between D2-like receptor binding and $\mathrm{AB}$ size, separately for the left (left panel) and the right (right panel) striatum. Binding values are based on the peak voxel within each striatal cluster. Non-ranked values are shown for illustrative purposes.

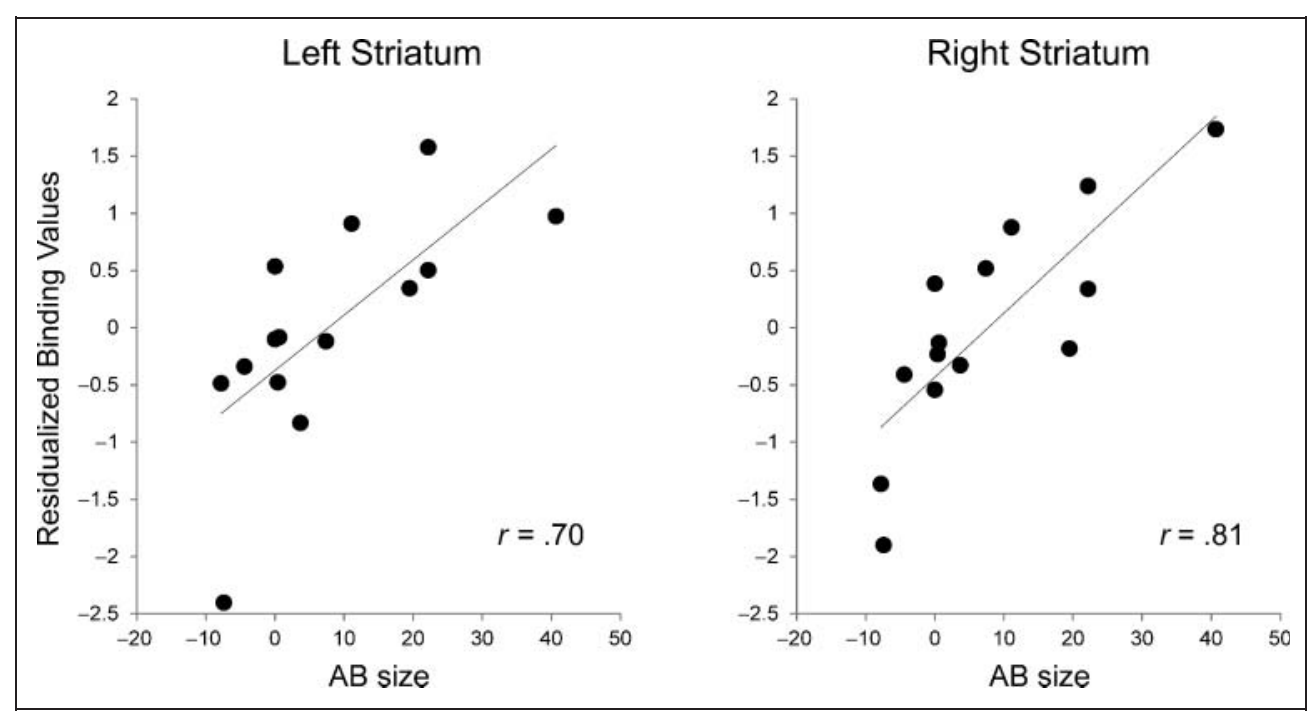


(Cools \& D'Esposito, 2011; Maia \& Frank, 2011). However, previous research also indicates that excessivly high striatal dopamine levels may impair performance as well, and it has been suggested that the relationship between cognitive performance and striatal dopamine level follows a U-shaped function (summarized in Cools \& D'Esposito, 2011), where both too little and too much striatal dopamine can hurt performance due to, respectively, impaired gating and inefficient gating (and contaminant distractibility). Our data, although indicative of a linear relationship, are not inconsisent with a $\mathrm{U}$ relationship between D2-like receptor binding and $\mathrm{AB}$ size, as it is conceivable that healthy subjects display a relatively restricted range in baseline dopamine level during resting conditions. On the basis of the larger literature on working memory and dopamine, we therefore hypothesize that striatal dopamine more likely exhibits a $U$ relationship with $A B$ size (see Figure 5), such that intermediate tonic dopamine levels promote optimal gating (or selective target updating) and hence a small $\mathrm{AB}$,whereas both excessively low and excessively high tonic dopamine levels produce a large $\mathrm{AB}$, albeit for different reasons. Whereas high dopamine levels will lead to more overall working memory updating and concomitant distractibility and a large $\mathrm{AB}$ (i.e., they also induce distracter updating, causing interference in working memory), very low levels will also be associated with a large $\mathrm{AB}$ due to a general poor updating ability (not enough dopamine to update even target information). Note that in the latter case in particular (general poor

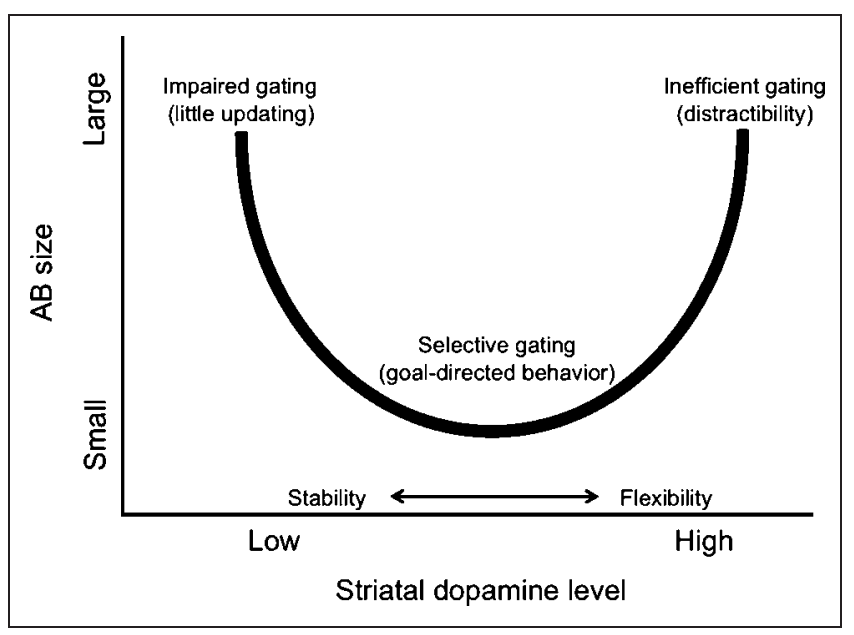

Figure 5. Hypothesized U relationship between tonic dopamine level and AB size. Dopamine level is hypothesized to determine the $\mathrm{AB}$ by regulating the threshold for working memory updating. Specifically, we postulate that $\mathrm{AB}$ size exhibits a $\mathrm{U}$ relationship with striatal dopamine level, such that (i) excessively high dopamine levels will result in inefficient gating (they also induce distracter updating, causing interference in working memory) and hence a large $\mathrm{AB}$, (ii) intermediate dopamine levels will promote optimal gating (selective target updating) and hence a small $\mathrm{AB}$, and (iii) very low levels will be associated with poor updating ability (not enough dopamine to update even target information) and both poor T1 accuracy and a large AB to T2. updating ability), one would also expect poor T1 accuracy. In the current study, with healthy individuals and $\mathrm{T} 1 \mathrm{accu}-$ racy at ceiling level (99-100\%), no relationship between D2-like binding and T1 accuracy was observed. Yet, it is of note that in the study by Colzato et al. (2008), the group of subjects with relatively low sEBR scores not only displayed a larger AB to T2 but also lower T1 accuracy compared with the group of subjects with relatively high sEBR scores. This observation is in line with the idea that low striatal dopamine levels lead to an overall impairment in updating ability. Yet, future research in which dopamine level is experimentally manipulated across a wide range is necessary to determine whether or not the relationship between $\mathrm{AB}$ size and dopamine level follows the proposed U-shaped function.

In summary, we hypothesize that striatal dopamine determines the $\mathrm{AB}$ by regulating the threshold for working memory updating. In this framework, the $\mathrm{AB}$ can be due both to a general poor updating ability, when the threshold for working updating is set too strongly (and striatal dopamine levels are very low), and to distracter interference in working memory, when the threshold for updating is set too lenient (and striatal dopamine levels are very high). This account, which highlights a neurobiological mechanism through which rapid input control might be accomplished, is in line with the notion that the $\mathrm{AB}$ results from online selection mechanisms that control information flow into working memory rather than being the result of T1-induced cognitive resource depletion (see also below). Yet, future studies, including pharmacological, patient, and neurocomputational studies, are necessary to determine the precise relationship (i.e., linear or U-shaped) between basal striatal dopamine activity and the ability to gate only newly task-relevant information into working memory under rapidly changing input conditions, such as the AB. This work should also address the relationship between tonic dopamine levels and phasic dopamine responses. Phasic dopamine in the striatum has been related to selective reinforcement of gating for task-relevant stimulation (e.g., Maia \& Frank, 2011), and large phasic responses may hence promote selective target updating in the AB task. Future work should also look into the possible role of striatal dopamine receptor features in the $\mathrm{AB}$. As pointed out above, D2-like receptor binding is proportional to both receptor density and endogenous dopamine, and on the basis of the current data alone, it is unclear to what extent the $\mathrm{AB}$ may be related to endogenous dopamine level, D2 receptor density, and/or other receptor features, such as affinity. Thus, more research is necessary for fine tuning the "dopamine hypothesis" of the AB and may ultimately lead to a mechanistic and neurobiologically grounded model of the AB.

It is noteworthy that the regions in which D2-like receptor binding predicted $\mathrm{AB}$ size fall within the associative striatum. This part of the striatum forms loops with associative regions of the cortex, such as dorsolateral pFC 
(Draganski et al., 2008; Joel \& Weiner, 2000). Notably, PET and fMRI studies have linked the associative striatum to flexible cognitive control, including operations such as working memory updating and inhibition of irrelevant information (e.g., Kühn et al., in press; Bäckman et al., 2011; Dahlin, Neely, Larsson, Bäckman, \& Nyberg, 2008; McNab \& Klingberg, 2008). Although striatal dopamine has been linked to many functions other than WM gating and our measure, D2-like receptor binding, hence does not provide a pure measure of WM gating, the location of our effects in the associative striatum is in line with our hypothesis that striatal dopamine determines the $\mathrm{AB}$ by regulating the threshold for WM updating.

It is of further note that the D2 receptor is expressed primarily in the striatal indirect pathway, which traverses the pallidum and subthalamic nucleus before targeting BG output structures (Gerfen, 1992). It has been posited that the striatum facilitates or suppresses cortical actions (e.g., working memory updating) via separate direct and indirect pathways, respectively, that project to frontal cortex and that the likelihood that a cognitive representation is selected for updating of working memory in the frontal cortex is proportional to the difference between activity for that representation in the direct and indirect pathways (Hazy, Frank, \& O'Reilly, 2007b). Indeed, the indirect pathway has previously been shown to play a critical role in filtering out distracting information from being updated into working memory (McNab \& Klingberg, 2008; Moustafa, Sherman, \& Frank, 2008; Frank \& O'Reilly, 2006; O'Reilly \& Frank, 2006). Given the critical role of the striatal D2/ indirect pathway in inhibiting distracting information, it is notable that recent behavioral studies have shown that individual differences in the ability to inhibit distracting information determine AB size (Arnell \& Stubitz, 2010; Martens \& Valchev, 2009; Dux \& Marois, 2008). In addition, the here-observed relationship between $\mathrm{D} 2$-like receptor binding and $\mathrm{AB}$ size may thus provide a neurobiological mechanism through which selection mechanisms control responses to distracting input and provide support for filter-based accounts, where the $\mathrm{AB}$ reflects the workings of a mechanism designed to filter out nonrelevant information (e.g., Olivers \& Meeter, 2008; Di Lollo et al., 2005; Raymond et al., 1992). One of these models (Olivers \& Meeter, 2008) actually postulated a possible role for the $B G$ in the $A B$ based on previous findings showing that a prefrontal/BG network regulates access to working memory (McNab \& Klingberg, 2008). Yet, our finding could be consistent with models that attribute the $\mathrm{AB}$ to the shielding of $\mathrm{T} 1$ processing as well (e.g., Taatgen, Juvina, Schipper, Borst, \& Martens, 2009; Wyble, Bowman, \& Nieuwenstein, 2009; Hommel et al., 2006; Jolicoeur \& Dell'Acqua, 1998). By preventing gating of irrelevant representations, striatal D2 receptors may also promote undisturbed consolidation of T1 in working memory. In either case, the observed relationship between D2like receptor binding and the $\mathrm{AB}$ suggests that mechanisms involved in regulating the contents of working memory and in particular mechanisms related to suppressing irrelevant information contribute significantly to this phenomenon. A challenge for current computational and theoretical accounts of the $\mathrm{AB}$ lies in connecting more directly with these neurobiological data. Our results also synthesize the $\mathrm{AB}$ literature with the largely separate literature associated with working memory and may thus in addition inform models of working memory that have not yet had to confront rapid presentation of stimuli (e.g., Hazy et al., 2007a; Frank et al., 2001) by providing information regarding the timing of mechanisms involved in gating of information.

A role for striatal dopamine in the $\mathrm{AB}$ also receives support, albeit more indirectly, from several previous studies. First, as mentioned in the introduction, Colzato and colleagues found that sEBR, a marker of striatal dopamine function (Karson, 1983), predicted individual $\mathrm{AB}$ size (Colzato et al., 2008) and, furthermore, that the C957T polymorphism at the DRD2 gene (associated with striatal $\mathrm{D} 2$ receptors) was associated with $\mathrm{AB}$ size whereas polymorphisms associated with frontal dopamine and norepinephrine were not (Colzato et al., 2011). In addition, people high in WM operation span-a measure associated with striatal dopaminergic activity (Cools, Gibbs, Miyakawa, Jagust, \& D'Esposito, 2008)—display a smaller AB (Colzato et al., 2007). Finally, Olivers and Nieuwenhuis (2006) reported a reduced $\mathrm{AB}$ when, before trial onset, participants viewed pictures of positive affective content, which are thought to stimulate the dopaminergic system (Ashby, Isen, \& Turken, 1999). Using PET imaging, the current data extend these findings by providing more direct evidence for a role of striatal dopamine and, specifically, the indirect striatal pathway in the AB. Future research in which striatal dopamine levels are manipulated pharmacologically is necessary to determine more precisely how striatal dopamine may control gating of rapidly changing visual input.

Yet, it should be noted that it is unlikely that any single neuromodulatory mechanism can explain the many experimental factors that are known to modulate the $\mathrm{AB}$. Other neurotransmitters and in particular norepinephrine, given its role in temporal attention (Aston-Jones \& Cohen, 2005), likely also contribute to this phenomenon. For example, whereas striatal dopamine may modulate the threshold for working memory updating, norephinephrine may enhance cortical target processing (Nieuwenhuis, Gilzenrat, Holmes, \& Cohen, 2005). Nevertheless, together with previous findings (Colzato et al., 2008, 2011), the current data suggest an important role for striatal dopamine and, specifically, the striatal indirect pathway in the $\mathrm{AB}$ and, more generally, in flexibly prioritizing relevant over irrelevant information.

\section{Acknowledgments}

This work was supported by the Netherlands Organization for Scientific Research (NWO VIDI grant to H. A. S., NWO VENI 
grant to L. S. C.) and a grant from the National Institute of Mental Health (MH43454 to R. J. D.). We thank two anonymous reviewers for their helpful comments.

Reprint requests should be sent to Heleen A. Slagter, Department of Psychology, University of Amsterdam, Weesperplein 4, 1018 XA Amsterdam, the Netherlands, or via e-mail: h.a.slagter@uva.nl.

\section{REFERENCES}

Arnell, K. M., Stokes, K. A., MacLean, M. H., \& Gicante, C. (2010). Executive control processes of working memory predict attentional blink magnitude over and above storage capacity. Psychological Research, 74, 1-11.

Arnell, K. M., \& Stubitz, S. M. (2010). Attentional blink magnitude is predicted by the ability to keep irrelevant material out of working memory. Psychological Research, 74, 457-467.

Ashby, F. G., Isen, A. M., \& Turken, A. U. (1999). A neuropsychological theory of positive affect and its influence on cognition. Psychological Review, 106, 529-550.

Aston-Jones, G., \& Cohen, J. D. (2005). An integrative theory of locus coeruleus-norepinephrine function: Adaptive gain and optimal performance. Annual Review of Neuroscience, 28, 403-450.

Bäckman, L., Nyberg, L., Soveri, A., Johansson, J., Andersson, M., Dahlin, E., et al. (2011). Effects of working-memory training on striatal dopamine release. Science, 333, 718.

Braver, T. S., \& Cohen, J. D. (1999). Dopamine, cognitive control, and schizophrenia: The gating model. Progress in Brain Research, 121, 327-349.

Chun, M. M., \& Potter, M. C. (1995). A two-stage model for multiple target detection in rapid serial visual presentation. Journal of Experimental Psychology: Human Perception and Performance, 21, 109-127.

Colzato, L. S., Slagter, H. A., de Rover, M., \& Hommel, B. (2011). Dopamine and the management of attentional resources: Genetic markers of striatal D2 dopamine predict individual differences in the attentional blink. Journal of Cognitive Neuroscience, 23, 3576-3585.

Colzato, L. S., Slagter, H. A., Spapé, M. M. A., \& Hommel, B. (2008). Blinks of the eye predict blinks of the mind. Neuropsychologia, 46, 3179-3183.

Colzato, L. S., Spapé, M., Pannebakker, M. M., \& Hommel, B. (2007). Working memory and the attentional blink: Blink size is predicted by individual differences in operation span. Psychonomic Bulletin \& Review, 14, 1051-1057.

Cools, R., \& D'Esposito, M. (2011). Inverted-U-shaped dopamine actions on human working memory and cognitive control. Biological Psychiatry, 69, e113-e125.

Cools, R., Gibbs, S. E., Miyakawa, A., Jagust, W., \& D’Esposito, M. (2008). Working memory capacity predicts dopamine synthesis capacity in the human striatum. The Journal of Neuroscience: The Official Journal of the Society for Neuroscience, 28, 1208-1212.

Dahlin, E., Neely, A. S., Larsson, A., Bäckman, L., \& Nyberg, L. (2008). Transfer of learning after updating training mediated by the striatum. Science, 320, 1510-1512.

Di Lollo, V., Kawahara, J., Shahab Ghorashi, S. M., \& Enns, J. T. (2005). The attentional blink: Resource depletion or temporary loss of control? Psychological Research, 69, 191-200.

Draganski, B., Kherif, F., Klöppel, S., Cook, P. A., Alexander, D. C., Parker, G. J. M., et al. (2008). Evidence for segregated and integrative connectivity patterns in the human basal ganglia. The Journal of Neuroscience: The Official Journal of the Society for Neuroscience, 28, 7143-7152.
Dux, P. E., \& Marois, R. (2008). Distractor inhibition predicts individual differences in the attentional blink. PloS One, 3, e3330.

Dux, P. E., \& Marois, R. (2009). The attentional blink: A review of data and theory. Attention, Perception $\&$ Psychophysics, 71, 1683-1700.

Frank, M. J., Loughry, B., \& O'Reilly, R. C. (2001). Interactions between frontal cortex and basal ganglia in working memory: A computational model. Cognitive, Affective \& Behavioral Neuroscience, 1, 137-160.

Frank, M. J., \& O'Reilly, R. C. (2006). A mechanistic account of striatal dopamine function in human cognition: Psychopharmacological studies with cabergoline and haloperidol. Behavioral Neuroscience, 120, 497-517.

Gerfen, C. R. (1992). The neostriatal mosaic: Multiple levels of compartmental organization. Journal of Neural Transmission. Supplementum, 36, 43-59.

Hazy, T. E., Frank, M. J., \& O’Reilly, R. C. (2007a). Towards an executive without a homunculus: Computational models of the prefrontal cortex/basal ganglia system. Philosophical Transactions of the Royal Society of London, Series B, Biological Sciences, 362, 1601-1613.

Hazy, T. E., Frank, M. J., \& O’Reilly, R. C. (2007b). Towards an executive without a homunculus: Computational models of the prefrontal cortex/basal ganglia system. Philosophical Transactions of the Royal Society of London, Series B, Biological Sciences, 362, 1601-1613.

Hommel, B., Kessler, K., Schmitz, F., Gross, J., Akyürek, E., Shapiro, K., et al. (2006). How the brain blinks: Towards a neurocognitive model of the attentional blink. Psychological Research, 70, 425-435.

Ichise, M., Toyama, H., Innis, R. B., \& Carson, R. E. (2002). Strategies to improve neuroreceptor parameter estimation by linear regression analysis. Journal of Cerebral Blood Flow and Metabolism: Official Journal of the International Society of Cerebral Blood Flow and Metabolism, 22, 1271-1281.

Innis, R. B., Cunningham, V. J., Delforge, J., Fujita, M., Gjedde, A., Gunn, R. N., et al. (2007). Consensus nomenclature for in vivo imaging of reversibly binding radioligands. Journal of Cerebral Blood Flow and Metabolism: Official Journal of the International Society of Cerebral Blood Flow and Metabolism, 27, 1533-1539.

Jenkinson, M., \& Smith, S. (2001). A global optimisation method for robust affine registration of brain images. Medical Image Analysis, 5, 143-156.

Joel, D., \& Weiner, I. (2000). The connections of the dopaminergic system with the striatum in rats and primates: An analysis with respect to the functional and compartmental organization of the striatum. Neuroscience, 96, 451-474.

Jolicoeur, P., \& Dell'Acqua, R. (1998). The demonstration of short-term consolidation. Cognitive Psychology, 36, 138-202.

Karson, C. N. (1983). Spontaneous eye-blink rates and dopaminergic systems. Brain: A Journal of Neurology, 106, 643-653.

Kawahara, J.-I., Kumada, T., \& Di Lollo, V. (2006). The attentional blink is governed by a temporary loss of control. Psychonomic Bulletin \& Review, 13, 886-890.

Kellendonk, C., Simpson, E. H., Polan, H. J., Malleret, G., Vronskaya, S., Winiger, V., et al. (2006). Transient and selective overexpression of dopamine D2 receptors in the striatum causes persistent abnormalities in prefrontal cortex functioning. Neuron, 49, 603-615.

Kühn, S., Schmiedek, F., Noack, H., Wenger, E., Bodammer, N. C., Lindenberger, U., et al. (in press). The dynamics of change in striatal activity following updating training. Human Brain Mapping. doi:10.1002/hbm.22007 
Logan, J., Fowler, J. S., Volkow, N. D., Wang, G. J., Ding, Y. S., \& Alexoff, D. L. (1996). Distribution volume ratios without blood sampling from graphical analysis of PET data. Journal of Cerebral Blood Flow and Metabolism: Official Journal of the International Society of Cerebral Blood Flow and Metabolism, 16, 834-840.

Maia, T. V., \& Frank, M. J. (2011). From reinforcement learning models to psychiatric and neurological disorders. Nature Neuroscience, 14, 154-162.

Martens, S., Munneke, J., Smid, H., \& Johnson, A. (2006). Quick minds don't blink: Electrophysiological correlates of individual differences in attentional selection. Journal of Cognitive Neuroscience, 18, 1423-1438.

Martens, S., \& Valchev, N. (2009). Individual differences in the attentional blink. The important role of irrelevant information. Experimental Psychology, 56, 18-26.

Martens, S., \& Wyble, B. (2010). The attentional blink: Past, present, and future of a blind spot in perceptual awareness. Neuroscience and Biobehavioral Reviews, 34, 947-957.

McNab, F., \& Klingberg, T. (2008). Prefrontal cortex and basal ganglia control access to working memory. Nature Neuroscience, 11, 103-107.

Moustafa, A. A., Sherman, S. J., \& Frank, M. J. (2008). A dopaminergic basis for working memory, learning and attentional shifting in Parkinsonism. Neuropsychologia, 46, 3144-3156.

Mukherjee, J., Christian, B. T., Dunigan, K. A., Shi, B., Narayanan, T. K., Satter, M., et al. (2002). Brain imaging of 18F-fallypride in normal volunteers: Blood analysis, distribution, test-retest studies, and preliminary assessment of sensitivity to aging effects on dopamine D-2/D-3 receptors. Synapse, 46, 170-188.

Mukherjee, J., Yang, Z. Y., Das, M. K., \& Brown, T. (1995). Fluorinated benzamide neuroleptics-III. Development of (S)-N-[(1-allyl-2-pyrrolidinyl)methyl]-5-(3-[18F] fluoropropyl)-2, 3-dimethoxybenzamide as an improved dopamine D-2 receptor tracer. Nuclear Medicine and Biology, 22, 283-296.

Nichols, T. E., \& Holmes, A. P. (2002). Nonparametric permutation tests for functional neuroimaging: A primer with examples. Human Brain Mapping, 15, 1-25.

Nieuwenhuis, S., Gilzenrat, M. S., Holmes, B. D., \& Cohen, J. D. (2005). The role of the locus coeruleus in mediating the attentional blink: A neurocomputational theory. Journal of Experimental Psychology: General, 134, 291-307.

Oakes, T. R., Fox, A. S., Johnstone, T., Chung, M. K., Kalin, N., \& Davidson, R. J. (2007). Integrating VBM into the general linear model with voxelwise anatomical covariates. Neuroimage, 34, 500-508.

Olivers, C. N. L., \& Meeter, M. (2008). A boost and bounce theory of temporal attention. Psychological Review, 115, 836-863.
Olivers, C. N. L., \& Nieuwenhuis, S. (2006). The beneficial effects of additional task load, positive affect, and instruction on the attentional blink. Journal of Experimental Psychology: Human Perception and Performance, 32, 364-379.

Olivers, C. N. L., van der Stigchel, S., \& Hulleman, J. (2007). Spreading the sparing: Against a limited-capacity account of the attentional blink. Psychological Research, 71, 126-139.

O'Reilly, R. C., \& Frank, M. J. (2006). Making working memory work: A computational model of learning in the prefrontal cortex and basal ganglia. Neural Computation, 18, 283-328.

Raymond, J. E., Shapiro, K. L., \& Arnell, K. M. (1992). Temporary suppression of visual processing in an RSVP task: An attentional blink? Journal of Experimental Psychology: Human Perception and Performance, 18, 849-860.

Shapiro, K. L., Raymond, J. E., \& Arnell, K. M. (1997). The attentional blink. Trends in Cognitive Sciences, 1, 291-296.

Slagter, H. A., Johnstone, T., Beets, I. A. M., \& Davidson, R. J. (2010). Neural competition for conscious representation across time: An fMRI study. PloS One, 5, e10556.

Slagter, H. A., Lutz, A., Greischar, L. L., Francis, A. D., Nieuwenhuis, S., Davis, J. M., et al. (2007). Mental training affects distribution of limited brain resources. PLoS Biology, 5, e138.

Slagter, H. A., Lutz, A., Greischar, L. L., Nieuwenhuis, S., \& Davidson, R. J. (2009). Theta phase synchrony and conscious target perception: Impact of intensive mental training. Journal of Cognitive Neuroscience, 21, 1536-1549.

Taatgen, N. A., Juvina, I., Schipper, M., Borst, J. P., \& Martens, S. (2009). Too much control can hurt: A threaded cognition model of the attentional blink. Cognitive Psychology, 59, $1-29$.

Wang, M., Vijayraghavan, S., \& Goldman-Rakic, P. S. (2004) Selective D2 receptor actions on the functional circuitry of working memory. Science, 303, 853-856.

Woodward, N. D., Zald, D. H., Ding, Z., Riccardi, P., Ansari, M. S., Baldwin, R. M., et al. (2009). Cerebral morphology and dopamine D2/D3 receptor distribution in humans: A combined [18F] fallypride and voxel-based morphometry study. Neuroimage, 46, 31-38.

Wyble, B., Bowman, H., \& Nieuwenstein, M. (2009). The attentional blink provides episodic distinctiveness: Sparing at a cost. Journal of Experimental Psychology: Human Perception and Performance, 35, 787-807.

Zhang, Y., Brady, M., \& Smith, S. (2001). Segmentation of brain MR images through a hidden Markov random field model and the expectation-maximization algorithm. IEEE Transactions on Medical Imaging, 20, 45-57. 